\title{
Deduction of Electric Field Module in a Multilayer of Isotropic Materials to Detect Surface Plasmons with a Graphical User Interface
}

\author{
B. Garibello ${ }^{1} \mathbb{D}$, Y. Martín $^{1}$ \\ ${ }^{1}$ Basic sciences department - Santo Tomás University, Cra. 9 \# 51-11, Bogotá, Colombia, \\ bernardogaribello@usantotomas.edu.co,juanamartin@usantotomas.edu.co.
}

\begin{abstract}
Electric field module for any isotropic multilayer thin film structure, is presented as analytical deduction. Analytic expressions for the electric field distribution are developed initially for a monolayer isotropic system based on Airy's formulae and boundary conditions, with an incident monochromatic source of light. The transfer matrix method $2 \times 2$, is used to deduce the distribution of the forward and backward electric field amplitudes on the inner layers in a general multilayer thin film structure. Analytical results are simulated in Transverse-Magnetic (TM) and Transverse-Electric (TE) modes making evident (when takes place) an electric field enhancement due to surface plasmons resonance. A graphical user interface is created to make steady simulations and create new structures as desired, minimizing time and optimizing resources.
\end{abstract}

Index Terms - Electric field, isotropy, multilayers, optical function, plasmons, resonance, transfer matrix.

\section{INTRODUCTION}

Light propagation over multilayer thin film structures have been widely studied in optics [1]- [2], applied physics [3] - [4], bio-sensing [5] - [6] and applied electromagnetism [7] - [8]. This article presents an analytical deduction of electric field module in multilayer systems of isotropic layered thin film media, a graphical user interface (GUI) is designed to plot optical functions of reflectance, transmittance and absorptance depending on the incidence angle of a monochromatic source of light. GUI also plot electric field module as function of transversal structure coordinate, assuming known the parameters: thicknesses of each layer, refractive index, and source wavelength. Initially, a monolayer system is analyzed, using the Airy's formulae [9] - [10], it will helped to plot optical functions in Kretschmann \& Raether geometry [11] whose results are consistent with data reported [12] . Optical functions were found from a general problem of propagation of light on isotropic multilayer systems using Yeh's matrix transfer method [13]. Optical functions are determined for Transverse-Electric (TE) and Transverse-Magnetic (TM) modes. Calculations of electric field module are made from the boundary conditions of electromagnetic fields on materials [14]. Optical functions 
Journal of Microwaves, Optoelectronics and Electromagnetic Applications, Vol. 20, No. 1, March 2021

DOI: http://dx.doi.org/10.1590/2179-10742021v20i1927

permit detects unusual absorptions of light on the structure and the special angle when it happens. In this angle, electric field distribution exhibit an amplitude enhancement associated to surface resonant plasmons present only in TM mode, this mode is also known as p-polarization. GUI designed, becomes relevant to perform an steady creation of multilayer thin film structures for optical functions (software related has developed in [15] - [16] ), but electric field distribution are not taking into account for each layer. Futhermore, designed computational tool makes possible handle parameters as: number of materials, inner layers thicknesses, and source wavelength. Some figures are included showing calculations for different multilayers and a general view of the user interface, concluding remarks shows how the software created help students to optimizing structures without wasting materials, helping save costs, resources and time.

\section{Proposed Methodology}

Development stages are mentioned below treating optical functions and deducing for a monolayer and multilayer structure, the electric field module from boundary conditions.

\section{A. Optical Functions and surface plasmons}

Optical functions of reflectance, transmittance and absorptance have been widely studied and simulated for multilayer thin film structures of isotropic materials. One of the most analyzed structures was created by Kretschmann \& Raether which have optical crystal (known as BK7) as incident medium, gold as inner layer and air as substrate. If there is a monochromatic incident source of light toward the structure with p-polarization, then, will be an unusual absorption of light at an specific angle [11]. The angle where this phenomenon takes place is called plasmon angle due to plasmonic resonances in the interface gold-air [17]. Plasmonic excitation can be achieved in two ways, from multilayer structures [18], or through nanoparticles [19]- [20]. On the first technique, there are Surface Plasmon Polaritons (SPP's), generated via thin film structures. The second one, nanoparticles are immerse into an external electric field generating Localized Surface Plasmons (LSP's) [21]- [22]. The treatment herein is only for the case of SPP's.

There is no way to do an experimental measure for electric (or magnetic) field, because of the inner layer thicknesses are in order of nanometers, so, the electric field enhancement due to plasmonic resonances could not be treated directly in laboratory for the TM mode. Analytical deduction and simulation of electric field distribution depending on the transversal coordinate of the structure, becomes useful to determinate how the plasmon resonance enhance the electric field amplitude. Electric field distribution on a multilayer system has been analyzed using matrix methods [23], and without using them [24]. 


\section{B. Monolayer Structures}

A monolayer structure is determined by a semi-infinite medium of incidence, an inner layer and a substrate considered as well semi-infinite. Kretschmann \& Raether geometry exhibits surface plasmon resonances for an specific angle, there is another geometry created by Andreas Otto [25], consisting of optical glass (BK7) as medium of incidence, air as inner layer and gold as substrate, this geometry also evidence resonance, but, is less analyzed because of its difficult experimental fabrication. Fig. 1, shows a schematic representation for Kretschmann \& Raether geometry, here, $k$ represents the wave vector, $k_{\chi}$ is the wave vector component where is an evanescent wave on the $X$-axis of the coordinate system established, $\theta$ is the angle of incidence respect to the normal. Plasmonic resonances in optic regime, for wavelength $X$-component [26] satisfies the dispersion equation (1):

$$
k_{x}=\frac{\omega}{C} \sqrt{\frac{\epsilon_{1} \epsilon_{2}}{\epsilon_{1}+\epsilon_{2}}}
$$

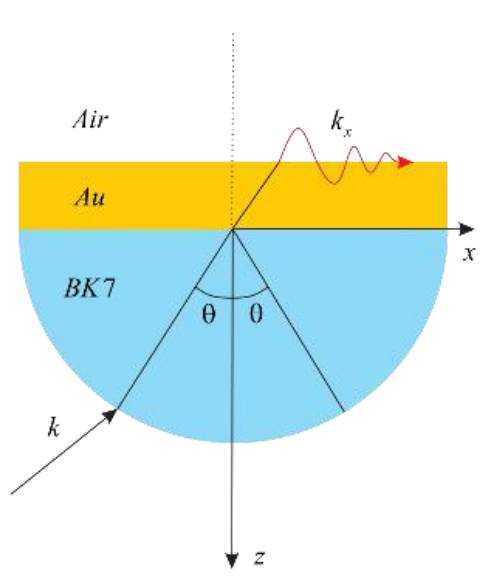

Fig. 1. Kretschmann \& Raether geometry for an arbitrary angle of incidence.

Here, $\epsilon_{1}$ and $\epsilon_{2}$ are the dielectric constants in this case for gold and air, respectively, $\omega$ is the angular frequency of the source and $c$ is the speed of light in vacuum.

In a general monolayer structure, the analysis developed by the astronomer George Bidell Airy (1.833) allows to find Fresnel coefficients [9] - [10], for a source of light coming from the incident medium, travelling along the three media (multiple reflections are considered) giving the equations (2) and (3) as follows:

$$
\begin{aligned}
& r=\frac{r_{12}+r_{23} e^{i \phi}}{1+r_{12} r_{23} e^{i \phi}} \\
& t=\frac{t_{12}+t_{23} e^{i \frac{1}{2} \phi}}{1+r_{12} r_{23} e^{i \phi}}
\end{aligned}
$$


Here $r$, is the Fresnel coefficient for reflection, $t$, is the Fresnel coefficient for transmission, $r_{12}$, is the Fresnel coefficient in the interface $1 \| 2, r_{23}$, is the Fresnel coefficient for interface $2 \| 3, t_{12}$ and $t_{23}$ have the same interpretation but, for transmission coefficients. The term $\phi=2 d \frac{\omega}{c} n_{2} \cos \theta_{2}$, is the phase shift, depending on the inner layer thickness $d$, angular frequency of the source $\omega$, speed of light in vacuum $C$, refractive index on the inner layer $n_{2}$ and transmission angle in the second medium $\theta_{2}$.

Analytical deductions are performed to find explicit expressions for electric field in the inner layer, expressions for magnetic field could be obtained using Maxwell equations. For s-polarization or TE mode, the electrical field is transverse (perpendicular) to the wave's incidence plane supposing there is no waves coming from the substrate, the incident reflected and transmitted waves are given by the equation (4):

$$
E_{y}(z)=\left\{\begin{array}{ccc}
A_{s} e^{i k_{1 z} z}+B_{s} e^{-i k_{1 z} z} & \text { si } & z \leq 0 \\
C_{s} e^{i k_{2 z} z}+D_{s} e^{-i k_{2 z} z} & \text { si } & 0<z<d \\
F_{s} e^{i k_{3 z} z} & \text { si } & z \geq d
\end{array}\right.
$$

Where $k_{i z}=\frac{\omega}{c} n_{i} \cos \theta_{i}$, for $i=1,2,3$. Complex constants $A_{s}, B_{s}, C_{s}, D_{s}, F_{s}$ are the forward and backward electric field amplitudes in each layer, refractive indexes are: $n_{1}, n_{2}, n_{3}$. It will assume, $A_{s}=1$ or a known amplitude defined by the source, so, $B_{s}=r_{s}$ and $F_{s}=t_{s}$, are Fresnel coefficients for reflection and transmission in s-polarization. Setting the parameters: materials refraction index, inner layer thickness and wavelength of the source, the goal herein is to find $C_{S}$ and $D_{s}$. Using boundary conditions [14], the parallel component of the electric field, must be continuous at the interfaces for $z=0$ and $z=d$, solving for $C_{s}$ and $D_{s}$ from the equation (4), are found explicit expressions in equations (5) and (6):

$$
\begin{array}{r}
C_{s}=\frac{t_{s} e^{i k_{2 z^{d}}}-\left(1+r_{s}\right)}{e^{2 i k_{2 z^{d}}}-1} \\
D_{s}=1+r_{s}-\frac{t_{s} e^{i k_{2 z} d}-\left(1+r_{s}\right)}{e^{2 i k_{2 z^{d}}}-1}
\end{array}
$$

In a similar way, for p-polarization, boundary condition for tangential continuous electric field is given by:

$$
E_{x}(z)=\left\{\begin{array}{ccc}
\left(A_{p} e^{i k_{1 z} z}+B_{p} e^{-i k_{1 z} z}\right) \cos \theta_{1} & \text { si } & z \leq 0 \\
\left(C_{p} e^{i k_{2 z} z}+D_{p} e^{-i k_{2 z} z}\right) \cos \theta_{2} & \text { si } & 0<z<d \\
\left(F_{p} e^{i k_{3 z} z}\right) \cos \theta_{3} & \text { si } & z \geq d
\end{array}\right.
$$


Performing similar algebraic steps, from equation (7), and solving for $C_{p}$ and $D_{p}$ (forward and backward) components of electric field, doing the same assumptions as s-polarization, is possible to get:

$$
\begin{array}{r}
C_{p}=\frac{\alpha_{12} \alpha_{23} t_{p} e^{i k_{2 z} d}-\left(1+r_{p}\right)}{\alpha_{12}\left(e^{2 i k_{2 z} d}-1\right)} \\
D_{p}=\frac{1+r_{p}}{\alpha_{12}}-\frac{\alpha_{12} \alpha_{23} t_{p} e^{i k_{2 z} d}-\left(1+r_{p}\right)}{\alpha_{12}\left(e^{2 i k_{2 z} d}-1\right)}
\end{array}
$$

Where $\alpha_{12}=\frac{\cos \theta_{2}}{\cos \theta_{1}}, \alpha_{23}=\frac{\cos \theta_{3}}{\cos \theta_{2}}$, by using Snell Law's equations (8) and (9) can be written as functions only of incidence angle $\theta_{1}$.

\section{Multilayer structures}

Henceforth, Yeh's deduction [13] is followed to obtain Fresnel coefficients and optical functions in multilayer thin film structures for isotropic materials. Anisotropic treatment for multilayers presented by Yeh has an $4 \times 4$ matrix transfer method, nevertheless, there is a singularity reports by [27] . In Fig. 2 there is a schematic graphic showing the medium of incidence of the light source, inner layers and substrate. All materials are labeled from 0 for incident media, to $N+1$ in the substrate, for a $N$ layers system. In Fig. 2, $n_{i}$ are the refractive index of each material, $A_{i}$ and $B_{i}$ represent forward and backward electric field amplitudes, for $i=0,1,2, \ldots N+1$ and $d_{j}$ will be thicknesses for inner layers, for $j=1,2, \ldots, N$.

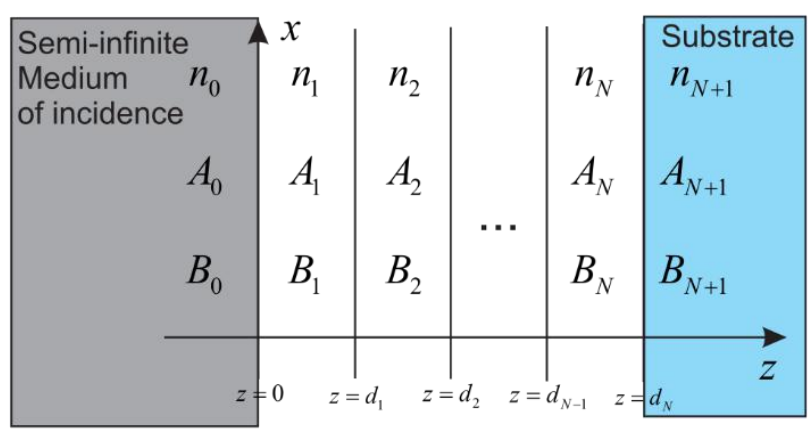

Fig. 2. Electric field backward and forward amplitudes in a multilayer thin film structure.

Transfer matrix method is compound by two kind of $2 \times 2$ matrices, dynamical matrix, and propagation matrix. Dynamical matrix is defined for $\mathrm{s}$ and p-polarization as shown in equations (10) and (11).

$$
D_{s}(i)=\left[\begin{array}{cc}
1 & 1 \\
n_{i} \cos \theta_{i} & -n_{i} \cos \theta_{i}
\end{array}\right]
$$




$$
D_{p}(i)=\left[\begin{array}{cc}
\cos \theta_{i} & \cos \theta_{i} \\
n_{i} & -n_{i}
\end{array}\right]
$$

Propagation matrix is defined for both polarizations as:

$$
P=\left[\begin{array}{cc}
e^{-i \frac{\phi}{2}} & 0 \\
0 & e^{i \frac{\phi}{2}}
\end{array}\right]
$$

If only two materials are involved, i.e., there is no inner layer, electric field amplitudes are obtained through dynamical matrices as:

$$
\left[\begin{array}{l}
A_{0} \\
B_{0}
\end{array}\right]=D_{0}^{-1} D_{1}\left[\begin{array}{l}
A_{1}^{\prime} \\
B_{1}^{\prime}
\end{array}\right]
$$

For all article purposes, $A_{0}$ (incident field amplitude) is assumed known, also, there is no wave coming from the substrate, this implies: $B_{1}^{\prime}=0$. Equation (13) is going to generate a couple of equations with a couple of unknowns $B_{0}$ and $A_{1}{ }^{\prime}$, for this case they will be Fresnel coefficients for reflection and transmission.

Electric field amplitudes become relevant from three media (one inner layer). In Fig. 3 a monolayer generic structure with incident (forward) and reflected (backward) electric field amplitudes, is shown.

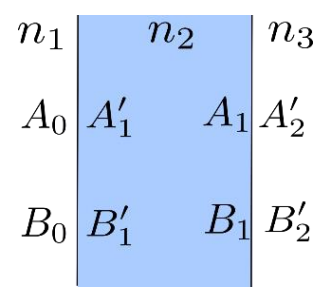

Fig. 3. Total incident and reflected electric field amplitudes in a monolayer structure.

Herein the electric field amplitudes forward and backward are $A_{1}$ and $B_{1}$. The phase shift will set the conditions on the amplitudes: $A_{1}=e^{-i \frac{\phi}{2}} A_{1}^{\prime}$ and $B_{1}=e^{-i \frac{\phi}{2}} B_{1}^{\prime}$. The matrix transfer method establish an expression involving matrices (10), (11) and (12), given by:

$$
\left[\begin{array}{l}
A_{1}^{\prime} \\
B_{1}^{\prime}
\end{array}\right]=P_{1} D_{1}^{-1} D_{2}\left[\begin{array}{l}
A_{2}^{\prime} \\
B_{2}^{\prime}
\end{array}\right]
$$

In presence of four materials there are two inner layers, will be six equations with six unknown quantities, in general for $N+2$ materials with $N$ layers, will be $2(N+1)$ equations with $2(N+$ 1) variables that are resoluble by the set of equations (15)-(18):

$$
\left[\begin{array}{l}
A_{0} \\
B_{0}
\end{array}\right]=D_{0}^{-1} D_{1}\left[\begin{array}{l}
A_{1}^{\prime} \\
B_{1}^{\prime}
\end{array}\right]
$$




$$
\begin{array}{r}
{\left[\begin{array}{l}
A_{1}^{\prime} \\
B_{1}^{\prime}
\end{array}\right]=P_{1} D_{1}^{-1} D_{2}\left[\begin{array}{l}
A_{2}^{\prime} \\
B_{2}^{\prime}
\end{array}\right]} \\
{\left[\begin{array}{l}
A_{2}^{\prime} \\
B_{2}^{\prime}
\end{array}\right]=P_{2} D_{2}^{-1} D_{3}\left[\begin{array}{l}
A_{3}^{\prime} \\
B_{3}^{\prime}
\end{array}\right]} \\
\vdots \\
{\left[\begin{array}{l}
A_{N}^{\prime} \\
B_{N}^{\prime}
\end{array}\right]=P_{N} D_{N}^{-1} D_{N}\left[\begin{array}{l}
A_{N+1}^{\prime} \\
B_{N+1}^{\prime}
\end{array}\right]}
\end{array}
$$

The $M$-Matrix relates amplitudes on incident medium with the amplitude on the substrate. This matrix is obtained with the thickness in each layer, the angle of incidence of the source of light and the wavelength of the incident beam, for both modes of propagation TE and TM, is given by:

$$
M=D_{0}^{-1}\left(\prod_{i=1}^{N} D_{i} P_{i} D_{i}^{-1}\right) D_{N+1}
$$

In addition, Fresnel coefficients are given by the equations (20) and (21):

$$
\begin{gathered}
r=\left(\frac{B_{0}}{A_{0}}\right)_{B_{N+1}^{\prime}=0} \\
t=\left(\frac{A_{N+1}^{\prime}}{A_{0}}\right)_{B_{N+1}^{\prime}=0}
\end{gathered}
$$

Using (20), $B_{0}$ is described by:

$$
r=\frac{B_{0}}{A_{0}}=\frac{M_{21}}{M_{11}} \rightarrow B_{0}=A_{0} \frac{M_{21}}{M_{11}}
$$

Doing a forward substitution, knowing the values $A_{0}$ and $B_{0}, A_{1}{ }^{\prime}$ and $B_{1}{ }^{\prime}$ are obtained replacing in (15). Having $A_{1}{ }^{\prime}$ and $B_{1}{ }^{\prime}, A_{2}{ }^{\prime}$ and $B_{2}{ }^{\prime}$ are found by the equation (16), and so on, for any number of layers. Since, previous procedure gives electric field total amplitudes in each layer, a plot as a function of the structure's transverse coordinate is capable of being programmed for all inner layers. For $i$-th layer, equation (23) is employed to calculate the total electric field:

$$
|\boldsymbol{E}(z)|=\left|A_{i} e^{i k_{i z}\left(z-d_{i}\right)}+B_{i} e^{i k_{i z}\left(z-d_{i}\right)}\right|
$$

\section{RESULTS AND DisCUSSION}

Simulations from theoretical expressions deduced in the preceding section will exhibit behaviour of optical functions and electric field module. First, optical functions for a monolayer known geometry Kretschmann \& Raether are calculated as so to achieve a plasmon angle in which calculate electric 
field module. In the same manner viz. the behaviour for a trilayer with a plasmon resonance is simulated.

\section{A. Kretschmann \& Raether optical functions and electric field module}

Optical functions for Kretschmann \& Raether geometry are plotted Fig. 4. The parameters for the structure are: refractive index for BK7: $n_{1}=1.51$, for gold: $n_{2}=0.183+3.43 i$ (here $i^{2}=-1$ ) and $n_{3}=1$ for the air, these refractive index are taken from the database created for Mikhail Polyanskiy [28]. Inner layer thickness is $d=47 \mathrm{~nm}$. The monochromatic source of light wavelength is $\lambda=633 \mathrm{~nm}$. There is an absolute wave absorption when the source makes an angle of $\theta_{p}=43.8^{\circ}$ (plasmon angle) respect to the normal. Fig. 4 can be contrasted with that obtained by Herreño [29], giving consistent results with the software designed.

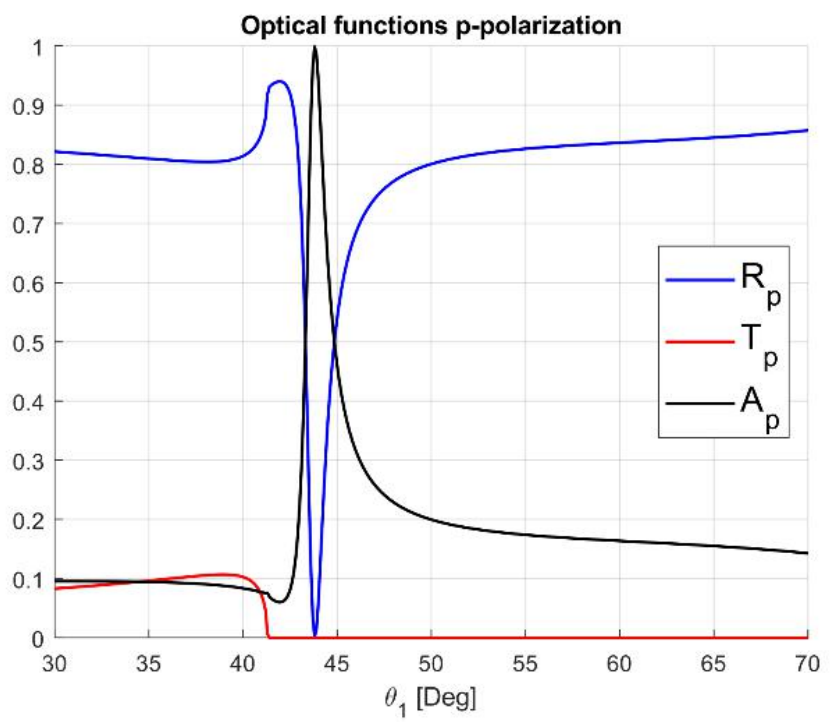

Fig. 4. Kretschmann \& Raether geometry optical functions for p-polarization.

The electric field module is represented for both polarizations (s and p) Fig. 5, $z$-axis corresponds to the thickness of gold, both functions are calculated in the plasmon angle, p-polarization (a) exhibits an increasing monotone behavior, due to resonance of the incident light beam with gold atoms at the interface with air [30], meanwhile (b) for s-polarization has a decreasing behavior. 


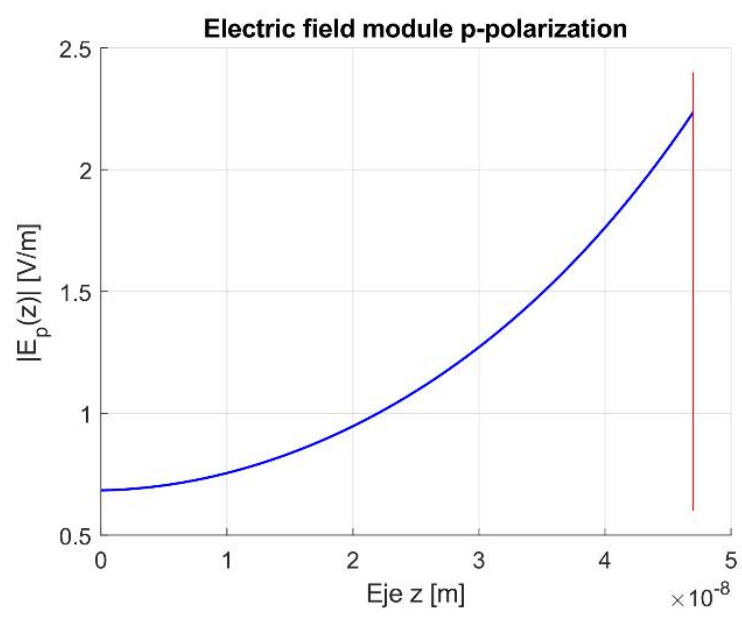

(a)

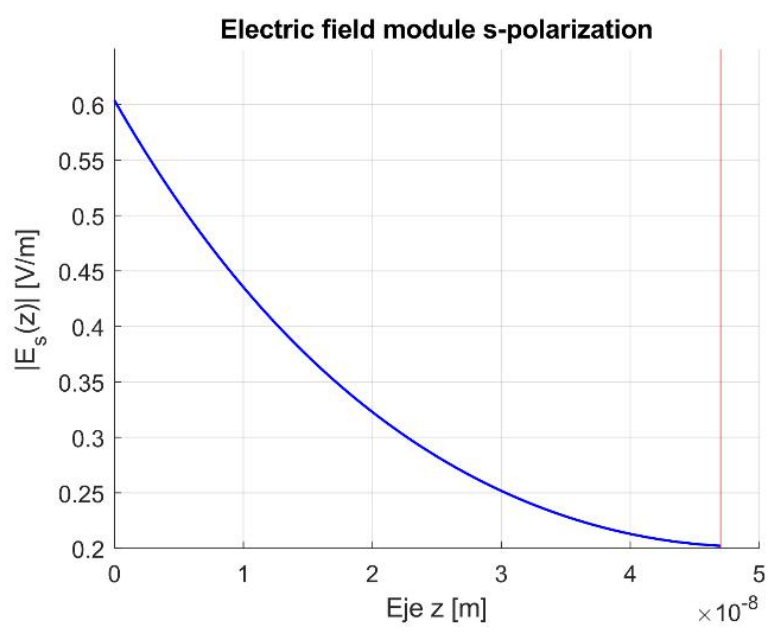

(b)

Fig. 5. Inner layer electric field distribution for TM and TE modes on the Kretschmann \& Raether geometry. Layer of gold has a thickness of $d=47 \mathrm{~nm}$. (a) Electric field module in the inner layer for p-polarization; (b) Electric field distribution in the inner layer for s-polarization.

\section{B. Trilayer Structure analysis}

Calculations are carried out for a Trilayer structure compound by BK7 as medium of incidence, $40 \mathrm{~nm}$ of gold, silicon dioxide $50 \mathrm{~nm}$, another layer of gold $5 \mathrm{~nm}$ and as a substrate air. A $\lambda=$ $633 \mathrm{~nm}$ monochromatic light beam is propagated towards the sample. The refractive indexes are: $n_{B K 7}=1.5151, n_{\text {Gold }}=0.18344+3.4332 i, n_{S i O_{2}}=1.457$ and $n_{\text {air }}=1$. Reflectance, transmittance and absorptance as function of the incident angle are plotted on Fig: 6. Angle where reflectance and transmittance go down to 0 is plasmon angle on $\theta_{p}=50^{\circ}$ there is a total wave absorption whereby, surface plasmon resonances are prestent. In addition, there is an angle for total internal reflection on $\theta=44.1^{\circ}$. 


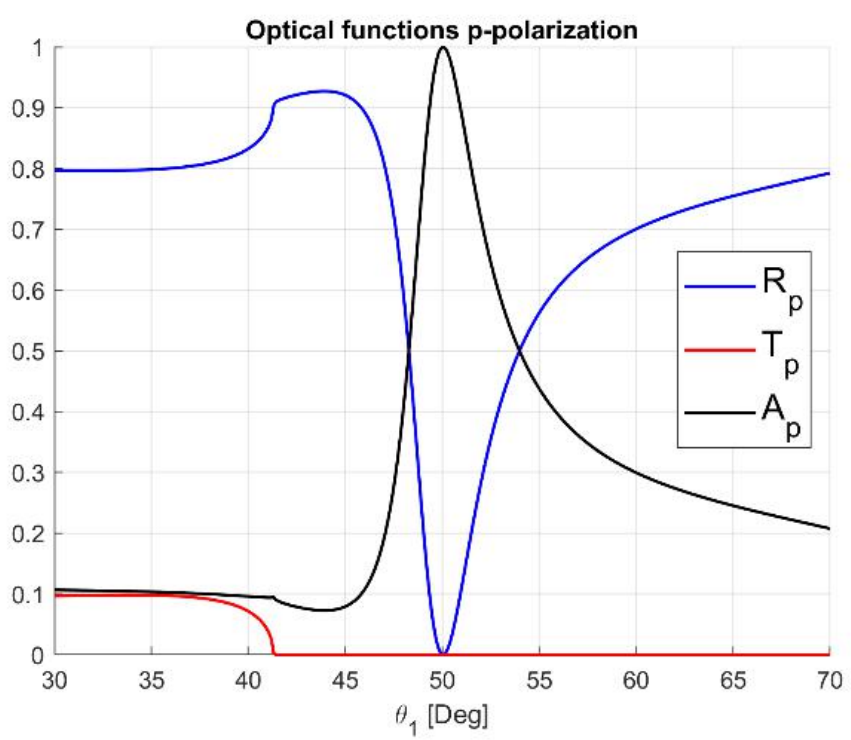

Fig. 6. Optical functions (reflectance, transmittance and absorptance) for a Trilayer geometry

$\left(B K 7\|A u\| \mathrm{SiO}_{2}\|\mathrm{Au}\| \mathrm{Air}\right.$ ) for p-polarization. Plasmon angle is present on $\theta_{p}=50^{\circ}$.

Electric field module is calculated on the inner layers of the structure, see Fig. 7. For p-polarization, there is a discontinuity on the change of each material, that is because of boundary condition for the normal electric field at interface in two media, while s-polarization does indicate a continuous curve, caused by the boundary condition for tangential electric field at interface of two media. Red vertical lines indicate change of material. Both graphics are simulated in plasmon angle. Note the enhancement for electric field for p-polarization (a), that is not present in the s-polarization (b).

Kretschmann \& Raether geometry is widely used on biosensing due to is possible set an organic sample in the air layer, so, reflectance curve slightly changes the resonant angle, this implies detection of urea and creatinine in solution of various concentrations [31], or molecular interactions [32] , alternatively gold can be changed by low cost materials as $\mathrm{Al}$ or $\mathrm{Al}_{2} \mathrm{O}_{3}$ having plasmonic resonances as well [33]. Multilayer structures simulated are the base in analysis in magneto-plasmonic structures as $\mathrm{Au}\|\mathrm{Co}\| \mathrm{Au}$ in which an external magnetic field (Transversal, longitudinal, and Polar) has shown an enhancing the magneto-optic Kerr effect [34]. 


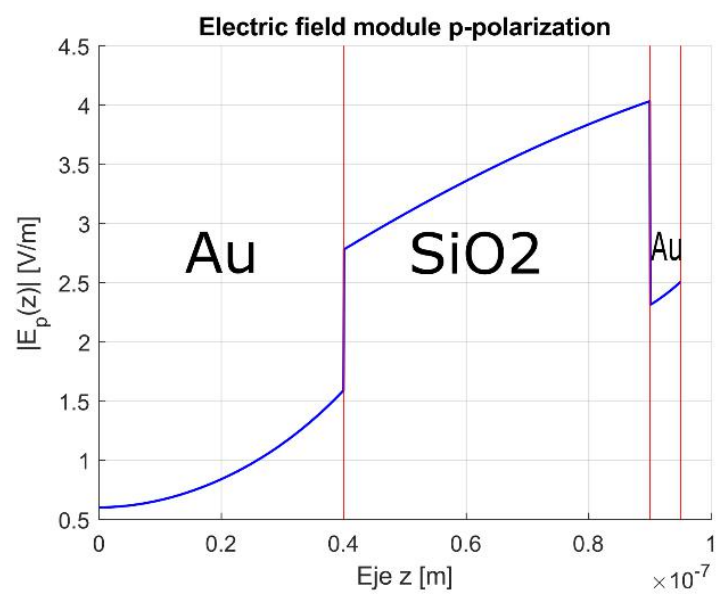

(a)

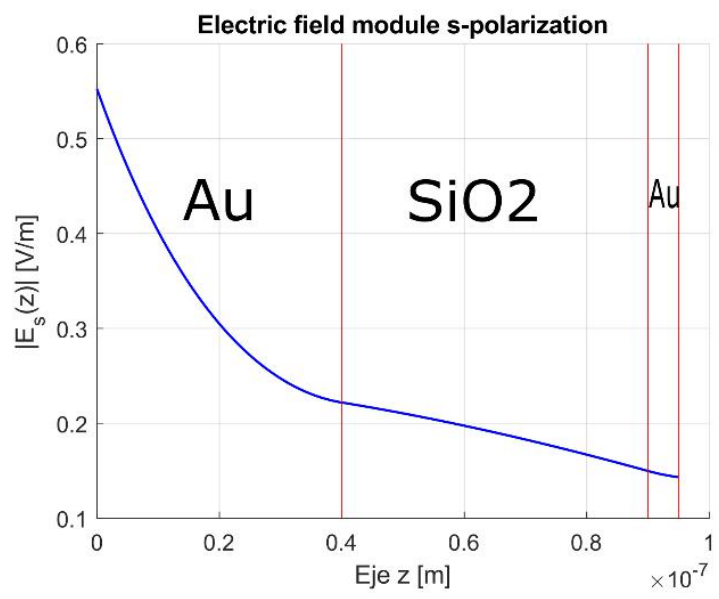

(b)

Fig. 7. Electric field distribution in the inner layer for $\mathrm{p}$ and s-polarizations for a Trilayer geometry

$\left(B K 7\|A u\| \mathrm{SiO}_{2}\|\mathrm{Au}\| \mathrm{Air}\right.$ ), calculated for plasmon angle on $\theta_{p}=50^{\circ}$. (a) Electric field module in the inner layers for p-polarization; (b) Electric field module in the inner layers for s-polarization.

\section{Graphical User Interface}

A graphical user interface involving all parameters for the multilayer thin film structure is usefulness in order to plot optical functions and electric field module [35]. The code was written on MATLAB, to run in any computer requires Runtime 8.5 or newest versions. It is possible to find similar software as the one developed by D. L. Windt [15] in which the primal approach is to find optical properties on multilayer films as reflectance or transmittance, more than to explore the presence of surface plasmons and produced enhancement of the electric field, analytical deduction for optical functions and electric field has other approach as presented here. Opti-layer software designed by A. Tikhonravov [16], is an alternative software that focus on reflectance, transmittance and ellipsometric aspects more than electric field or surface plasmons. 
Designed software have four panel described as follows; first, the left-upper panel contains input parameters: number of materials, refractive indexes, inner layer thicknesses and source of light wavelength. There is a couple of buttons that allows the user to create a simulation for TM mode (ppolarization) or TE mode (s-polarization). Moreover, there are three check boxes where user choose the optical function to visualize. The second left-down panel, is axis for plotting optical functions depending on the angle of incidence, on layered media when reflectance and absorptance are zero for an specific angle there are plasmonic resonances (always on the interface of a dielectric with a noble metal [36] - [37]). On the third right-upper panel is set the angle for calculate electric field module associated with the structure introduced on the left, there are couple of buttons to select which polarization to plot. The fourth right-down panel is the axis where is plotted the electric field module in each of the inner layers, as can be seen in Fig. 8.

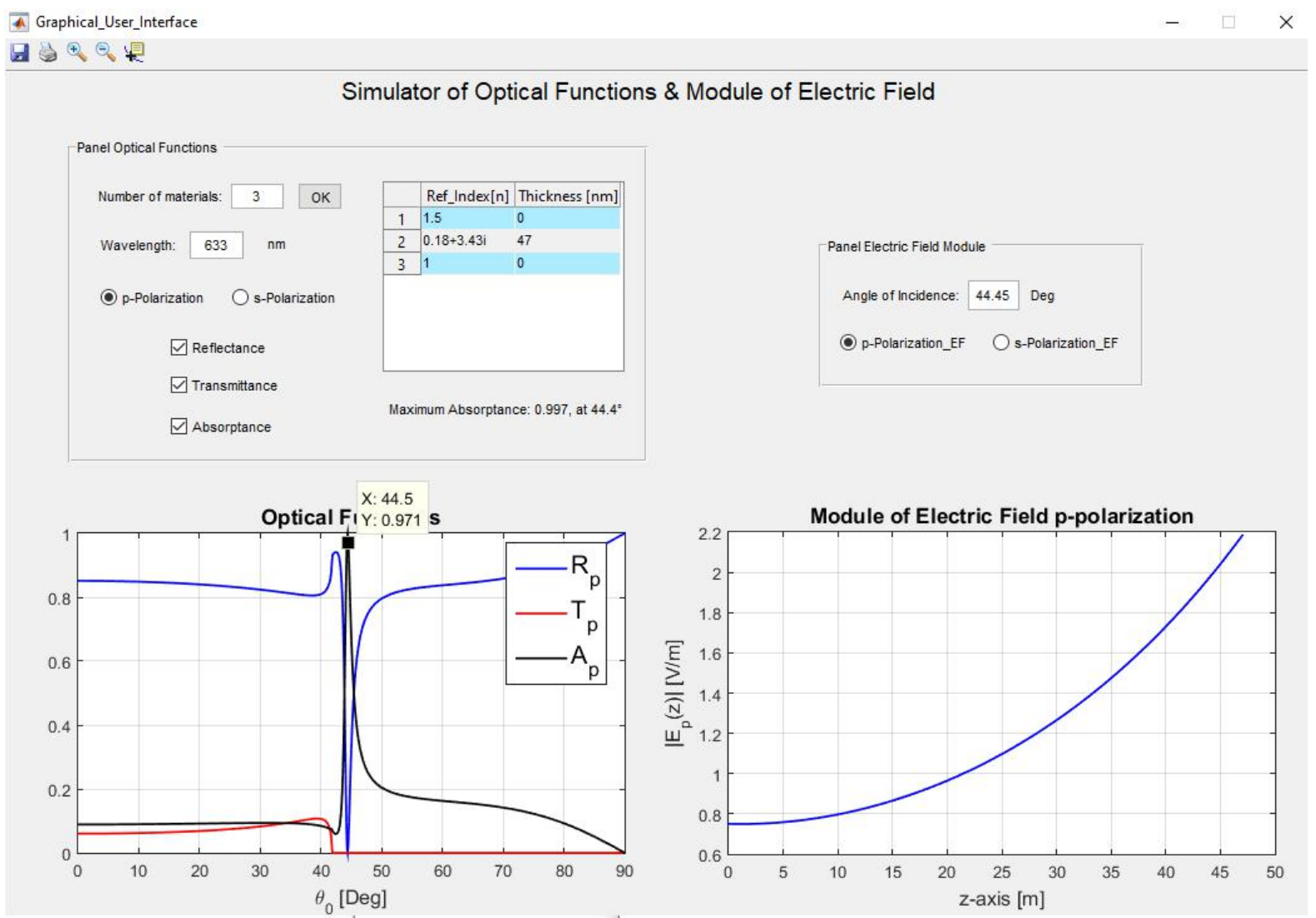

Fig. 8. GUI designed for optical functions and distribution of electric field.

A couple of flux diagrams of back end programming for optical functions and electric field distribution are shown in figures Fig. 9 and Fig. 10. 


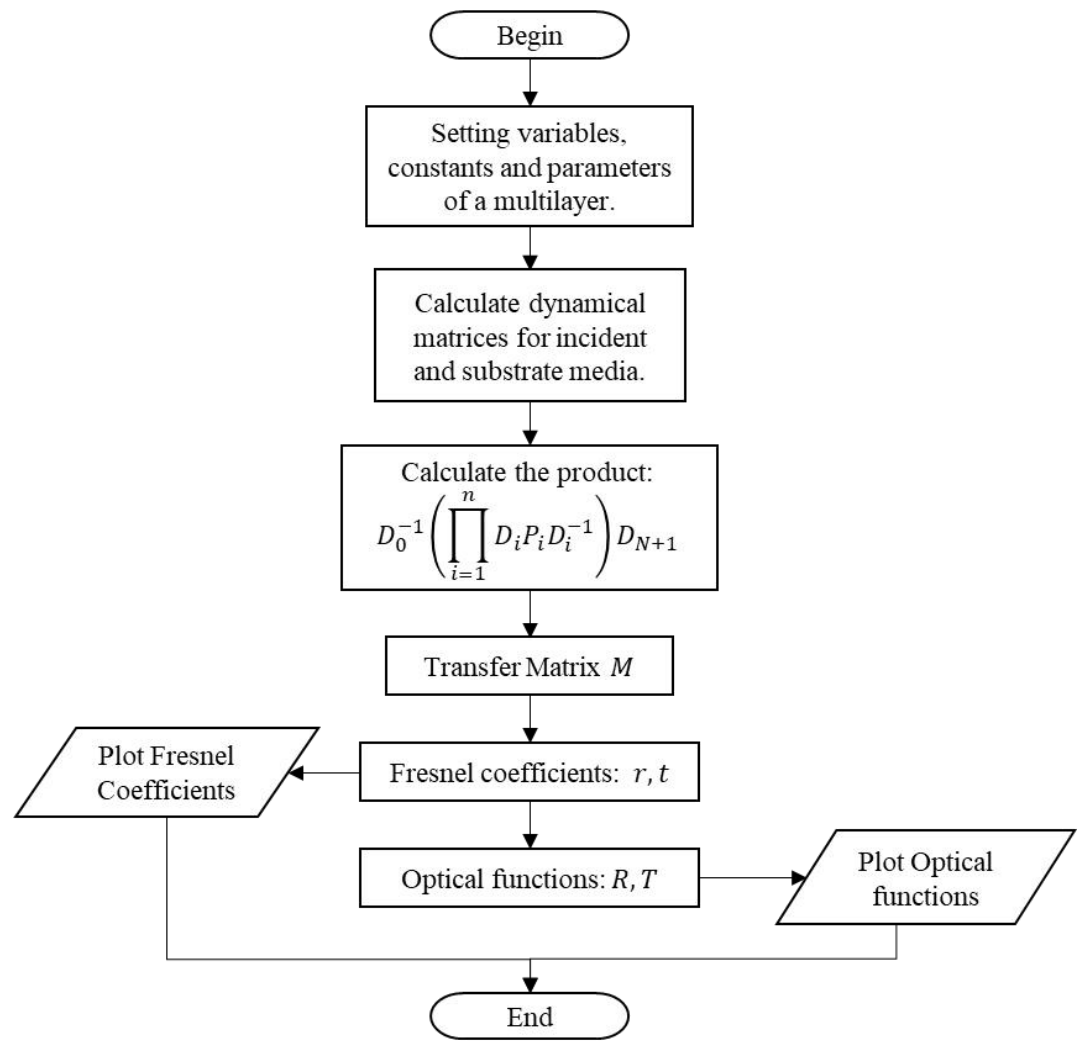

Fig. 9. Flux diagram to plot Fresnel coefficients and optical functions using transfer matrix method.

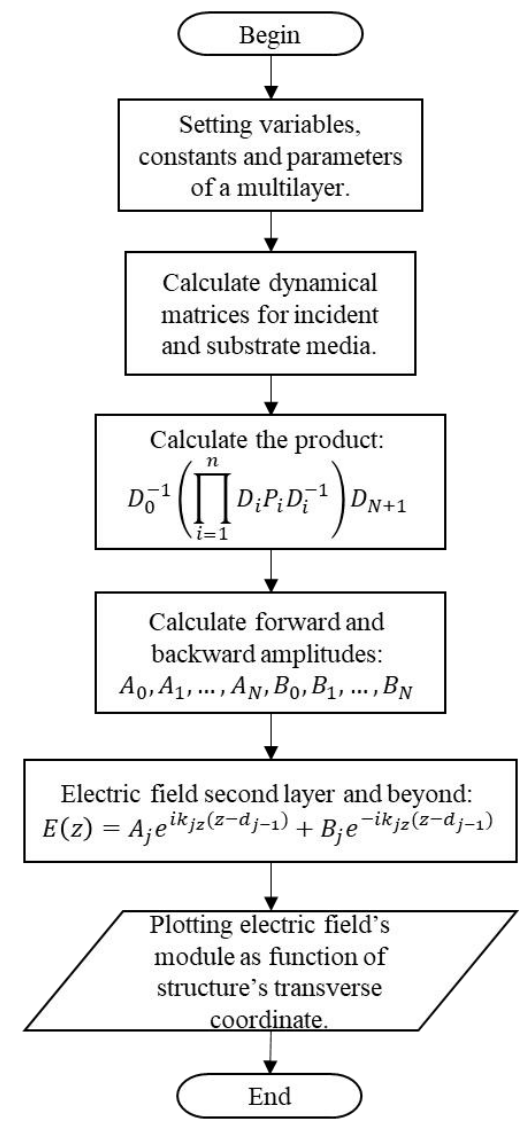

Fig. 10. Flux diagram to plot electric field module using transfer matrix method. 


\section{CONCLUDing ReMARKS}

On this paper was carried out the analytical deductions for total electric field module for monolayer system, based on the Airy's equations. The general case, for propagation of light on isotropic multilayer thin film structure, $2 \times 2$ transfer matrix method is used, and electric field amplitudes is derived in each layer. When surface plasmons resonances (unusual absorptions) are involved in multilayer structures there is an enhancement of the electric field, present only in p-polarization impossible to measure in a laboratory because of the dimensions of the structure.

Computational tool created has made possible to calculate optical functions and electric field module, for an arbitrary number of layers, saving time, money, and resources, creating experimental structures on lab only when the structure has been optimized via software. It is essential to note, that the GUI was register in Dirección Nacional de Derechos de Autor, Bogotá, Colombia, recognized as unpublished work.

Finally, is important to exalt that calculations derived from the software were useful in the develop of thesis: Evaluación del límite de detección y sensibilidad de detectores basados en resonancias plasmónicas, Optimización de la resonancia plasmónica en multicapas $\mathrm{Au}\left\|\mathrm{SiO}_{2}\right\| \mathrm{Au}$ and Control de la respuesta óptica efectiva de multicapas mediante la excitación de diferentes modos plasmónicos. Whose results were tested in lab showing consistence with theoretical and simulations framework presented.

\section{ACKNOWLEDGEMENT}

This project was supported by Universidad Santo Tomás de Aquino, Bogotá, giving the time to the authors to design the GUI (Front end) and its code (Back end).

\section{REFERENCES}

[1] M. Born and E. Wolf, Principles of optics: electromagnetic theory of propagation, interference and diffraction of light, Elsevier, 2013, pp. 51-70.

[2] O. Heavens, Optical properties of thin solid films, Courier Corporation, 1991, pp. 46-73.

[3] E. M. Schmidlin and H. J. Simon, "Observation of long range surface plasmon decay length by optical second harmonic generation," Applied optics, vol. 28, no. 16, pp. 3323 - 3326, 1989.

[4] P. Martinot, S. Laval and A. Koster, "Optical bistability from surface plasmon excitation through a nonlinear medium," Journal de Physique, vol. 45, no. 3, pp. 597 - 600, 1984.

[5] V. Shpacovitch and R. Hergenröder, "Surface Plasmon Resonance (SPR)-Based Biosensors as Instruments with High Versatility and Sensitivity," Multidisciplinary Digital Publishing Institute, 2020.

[6] B. Sepúlveda, A. Calle, L. M. Lechuga y G. Armelles, «Highly sensitive detection of biomolecules with the magnetooptic surface-plasmon-resonance sensor,» Opt. Let., vol. 31, nº 8, pp. 1085-1087, 2006. doi: 10.1364/OL.31.001085.

[7] D. Ferreira, A. Tinoco Salazar, I. Bianchi and . J. d. S. Lacava, "Planar multilayer structure analysis: an educational approach," J. of Microwaves, Optoelectronics and Electromagnetic Applications, vol. 11, no. 1, pp. 93 - 106, 2012.

[8] M. Africano, J. O. Vargas, R. Adriano, Oliveira and A. C. Lisboa, "Ground-Penetrating Radar Antenna Design for Homogeneous and Low-Loss Dielectric Multilayer Media," Journal of Microwaves, Optoelectronics and Electromagnetic Applications, vol. 19, no. 2, pp. 137 - 151, 2020.

[9] J. Reitz, F. Milford and R. Christy, Foundations of Electromagnetic Theory, USA: Addison-Wesley Publishing Company, 2008, pp. 441-466. 
[10] H. Fujiwara, Spectroscopic ellipsometry: principles and applications, John Wiley \& Sons, 2007, pp. 32-47.

[11] H. Raether, "Surface plasmons on smooth surfaces," in Surface plasmons on smooth and rough surfaces and on gratings, Springer, 1988, pp. 4-39. doi: 10.1007/BFb0048319.

[12] Y. Akimov, M. E. Pam and S. Sun, "Kretschmann-Raether configuration: Revision of the theory of resonant interaction," Physical Review B, vol. 96, no. 15, p. 155433, 2017.

[13] P. Yeh, Optical Waves in Layered Media, California: John Wiley \& Sons, 2005, pp. 102-114.

[14] D. J. Griffiths, Introduction to Electrodynamics, Pearson, Addison-Wesley, 2013.

[15] D. L. Windt, "IMD—Software for modeling the optical properties of multilayer films," Computers in physics, vol. 12, no. 4, pp. 360-370, 1998. doi: 10.1063/1.168689.

[16] A. Tikhonravov, "OptiLayer thin film soft-ware," OptiLayer GmbH, [Online]. Available: https://www.optilayer.com/. [Accessed 1606 2020].

[17] H. R. Gwon and S. H. Lee, "Spectral and angular responses of surface plasmon resonance based on the Kretschmann prism configuration," Materials transactions, vol. 51, no. 6, pp. 1150-1155, 2010. doi: 10.2320/matertrans.M2010003.

[18] J. R. Sambles, G. W. Bradbery and F. Yang, "Optical excitation of surface plasmons: an introduction," Contemporary physics, vol. 32, no. 3, pp. 173-183, 1991. doi: 10.1080/00107519108211048.

[19] E. Hutter and J. H. Fendler, "Exploitation of localized surface plasmon resonance," Advanced materials, vol. 16, no. 19, pp. 1685-1706, 2004. doi: 10.1002/adma.200400271.

[20] K. A. Willets and R. P. Van Duyne, "Localized surface plasmon resonance spectroscopy and sensing," Annu. Rev. Phys. Chem., vol. 58, pp. 267-297, 2007. doi: 10.1146/annurev.physchem.58.032806.104607.

[21] G. Armelles, A. Cebollada, A. García-Martín and M. U. Gonzalez, "Magnetoplasmonics: combining magnetic and plasmonic functionalities.," Advanced Optical Materials, vol. 1, no. 1, pp. 10-35, 2013. doi: 10.1002/adom.201200011.

[22] F. J. García Vidal and L. Martín Moreno, "Plasmones superficiales," 2008. [Online]. Available: https://www.investigacionyciencia.es/files/3055.pdf. [Accessed 11 abril 2020].

[23] A. Shalabney and I. Abdulhalim, "Electromagnetic fields distribution in multilayer thin film structures and the origin of sensitivity enhancement in surface plasmon resonance sensors," Sensors and Actuators A: Physical, vol. 159, no. 1, pp. 24-32, 2010. doi: 10.1016/j.sna.2010.02.005.

[24] B. Royuk, A calculation of electric field strengths for light in a multilayer thin film structure, Illinois: Master's Thesis. Southern Illinois University., 1996.

[25] A. Otto, "Excitation of nonradiative surface plasma waves in silver by the method of frustrated total reflection," Zeitschrift für Physik A Hadrons and nuclei, vol. 216, no. 4, pp. 398-410, 1968. doi: 10.1007/BF01391532.

[26] R. B. Schasfoort, Handbook of Surface Plasmon Resonance, United Kingdom: Royal Soc. of Chem., 2017, pp. 33-34.

[27] B. Garibello, N. Avilán, J. A. Galvis and C. A. Herreño-Fierro, "On the singularity of the Yeh $4 \times 4$ transfer matrix formalism," Journal of Modern Optics, https://doi.org/10.1080/09500340.2020.1775905, pp. 1-5, 2020.

[28] M. Polyanskiy, "Refractive Index database," 2008 - 2020. [Online]. Available: https://refractiveindex.info/. [Accessed $11042020]$.

[29] C. A. H. Fierro, Magnetoplasmónica de estructuras multicapa AulColAu, Bogotá: Universidad de los Andes, 2016.

[30] C. Hermann, V. A. Kosobukin, G. Lampel, J. Peretti, V. I. Safarov and P. Bertrand, "Surface-enhanced magnetooptics in metallic multilayer films," Physical Review B, vol. 64, no. 23, p. 235422, 2001. doi: 10.1103/PhysRevB.64.235422.

[31] P. S. Menon, F. A. Said, G. S. Mei, D. D. Berhanuddin, A. A. Umar, S. M. Shaari and Y. Burhanuddin, "Urea and creatinine detection on nano-laminated gold thin film using Kretschmann-based surface plasmon resonance biosensor," PLoS One, vol. 13, no. 7, p. e0201228, 2018.

[32] R. Borghol and T. Aguili, "Modeling of surface plasmon resonance nanobiosensor with the transverse resonance method," International Journal of RF and Microwave Computer-Aided Engineering, vol. 29, no. 8, p. e21792, 2019.

[33] A. S. Lambert, S. N. Valiulis, A. S. Malinick, I. Tanabe and Q. Cheng, "Plasmonic Biosensing with Aluminum Thin Films under the Kretschmann Configuration," Analytical Chemistry, 2020.

[34] C. A. Herreño Fierro and E. J. Patiño, "Maximization of surface-enhanced transversal magneto-optic Kerr effect in $\mathrm{Au} / \mathrm{Co} / \mathrm{Au}$ thin films," physica status solidi (b), vol. 252, no. 2, pp. 316-322, 2015.

[35] B. Garibello and Y. Martín, "Simulación de las funciones ópticas y detección en plasones de superficie en multicapas isotrópicas," in Memorias congreso internacional de ciencias básicas e ingeniería. CICI. ISBN: 978-958-8927-45-9, Villaviencio, 2018.

[36] R. Zia, M. Selker, P. Catrysse and M. Brongersma, "Geometries and materials for subwavelength surface plasmon modes," JOSA A, vol. 21, no. 12, pp. 2442 - 2446, 2004.

[37] A. Zayats, I. I. Smolyaninov and A. A. Maradudin, "Nano-optics of surface plasmon polaritons," Physics reports, vol. 408, no. 3 - 4, pp. $131-314,2005$ 